\title{
Combining Interaction and Automation in Process Algebra Verification *
}

\author{
Albert Camilleri \\ Hewlett-Packard Laboratories \\ Filton Road, Stoke Gifford, Bristol BS12 6QZ, U. K. \\ Paola Inverardi \\ Istituto di Elaborazione dell' Informazione, C. N. R. \\ Via Santa Maria 46, I-56100 Pisa, Italy. \\ Monica Nesi \\ University of Cambridge, Computer Laboratory \\ New Museums Site, Pembroke Street, Cambridge CB2 3QG, U. K.
}

\begin{abstract}
Most existing verification tools for process algebras allow the correctness of specifications to be checked in a fully automatic fashion. These systems have the obvious advantage of being easy to use, but unfortunately they also have some drawbacks. In particular, they do not always succeed in completing the verification analysis, due to the problem of state explosion, and they do not provide any insight into the meaning of the intended specifications. In this paper we consider an alternative approach in which both interactive and automatic techniques are combined in the hope that the advantages of automation are retained, and that some of its disadvantages are overcome. To achieve our goal, we use the interactive theorem prover HOL as a framework for supporting the theory of observational congruence of CCS, and provide a set of automatic proof tools, based on the algebraic axiomatization of the language, which can be used interactively. To illustrate how interaction and automation can be intermixed, we describe two verification strategies which exhibit different degrees of user interaction.
\end{abstract}

\section{Introduction}

In the past few years, many verification tools based on process algebras (e.g. CCS, CSP, ACP, LOTOS) have been proposed for proving properties of concurrent systems. Most of them generate a state transition representation of process algebra specifications, and use these to verify equivalences of specifications, and to show that specifications satisfy some logical (modal) properties. All these systems function fully automatically, and no facility is provided to perform any incremental or interactive reasoning.

Our experience is that it is not always possible to rely on a completely automatic approach to verification, even in the simple case of finite processes. Apart from being necessary to address problems which are, in the general case, undecidable, a system which leaves some crucial decisions to the user can, for efficiency reasons, also be useful when tackling decidable problems. A totally

\footnotetext{
"Research supported by, and collaboratively carried out at, Hewlett-Packard Laboratories, Pisa Science Centre, Corso Italia 115, I-56125 Pisa, Italy.
} 
interactive system, of course, will not be practical, but if an interactive approach can be used in conjunction with some automatic tools, then significant benefits can be gained. For example, another advantage of interactive reasoning is that it allows for a better understanding of the specifications, and of the correctness criteria one is attempting to verify.

In this paper, we describe ongoing work on an interactive verification environment for CCs specifications [23] based on the HOL theorem prover [15]. We show how the formal theory for a specific semantics of CCS, namely observational congruence, can be represented in the logic, and how the resulting representation can be the basis for higher level verification strategies by mechanized formal proof.

This work integrates, and further develops, two separate approaches in process algebra verification, namely $[10,2]$, which, although originated in different contexts, were based on the same basic ideas. Our approach is to exploit the algebraic nature of the axiomatization of CCS, and to allow the user to steer and control the verification process. For this we propose that the system offers functionalities to perform proofs automatically, but with the possibility to interact when automation is not desirable. Although we only discuss this approach in relation to the theory of observational congruence of cCs, it should theoretically provide a natural and flexible way for mechanizing different process algebras, for supporting alternative semantics of the same algebra, and for soundly extending semantic models to provide additional concepts.

In the following, we first give a brief description of the Hol theorem prover and its logic. Then, we introduce the subset of the CCS language under consideration, and show how the syntactic definitions and axiomatic characterization of the observational semantics of this subset of ccs can be incorporated in HOL. Next, we illustrate how reasoning about CCS specifications can be done in the resulting framework, by describing two verification strategies for use with different degrees of interaction. We then apply one of these strategies to prove a correctness criteria for a simple scheduler, and finally, discuss related work and possible extensions to the described research.

\section{The HOL System}

Higher order logic is a good formalism for mechanizing other mathematical languages because it is both powerful and general enough to allow sound and practical formulations. It has been used to express several other logics and process algebras [16,3], such as Floyd-Hoare logic, temporal and modal logics, vDM-style specifications, Dijkstra's weakest preconditions and CSP.

The theorem proving system used in the mechanizations described in $[3,16]$ is called HOL [15]. The HOL system, developed by Gordon, is based directly on the LCF theorem prover [24], and the theorem proving methodology supported by HOL is inherited from that originally developed by Milner for LCF. Below, we describe briefly the variety of higher order logic supported by HOL and the way in which it is manipulated to conduct proofs.

\subsection{The HOL Logic}

The HoL logic is a variety of higher order logic based on Church's formulation of Type Theory [4]. In the HOL logic, one uses standard predicate logic notation: $P x$ to express the proposition that $x$ has the property $P, R(x, y)$ to assert that the relation $R$ holds between $x$ and $y$, and the $\log$ ical connectives for constructing propositions $\neg, \wedge, \vee, \supset$ and $\equiv$ to express negation, conjunction, disjunction, implication and equivalence respectively. The conventional notation of universal and existential quantifiers for binding free variables is also used: $\forall x . P x$ to express the assertion that $P$ holds for every value of $x$, and $\exists x . P x$ to express the assertion that $P$ holds for at least one value of $x$. Additional notation includes $b \Rightarrow t_{1} \mid t_{2}$, which is used to represent the conditional 'if $b$ then $t_{1}$ else $t_{2}^{\prime}$. 
Higher order logic extends the above predicate calculus notation in the following ways:

- variables are allowed to range over functions, and the arguments of functions can themselves be functions (hence higher order),

- functions can be written as $\lambda$-abstractions,

- terms can be polymorphic, i.e. terms can have variable types (every term in the logic must be typed, to avoid Russell's paradox [15]), and

- Hilbert's choice operator, $\varepsilon$, is included in the logic.

\subsection{The HOL Theorem Prover}

The logic described above is mechanized using the general purpose programming language ML [7]. Terms of the HOL logic are distinguished from other ML expressions by being assigned the ML type term. Every HOL expression must be well typed according to the typing rules of the logic before being accepted by ML as a value of type term.

The ML language is used to manipulate HOL logic terms. In particular, ML is used to prove that certain terms are theorems. Theorems proved in the system are distinguished from ordinary terms by being assigned another built-in ML type thm. A theorem is represented by a finite set of terms called assumptions and a term called a conclusion. Given a set of assumptions $\Gamma$ and a conclusion $t$, we write $\Gamma \vdash t$ to represent the corresponding theorem or, if $\Gamma$ is empty, $\vdash t$. To introduce values of type $t h m$ into $\mathrm{ML}$, they must either be postulated as axioms or deduced from existing theorems by ML programs called inference rules.

Certain kinds of axioms are classed as definitions. These are axioms of the form $t c=t$ where $c$ is a constant not previously defined and $t$ is a term containing no free variables. Ideally, all axioms should be of this definitional form, since the freedom to postulate arbitrary axioms allows the danger of introducing inconsistencies. It is safe to write axioms in this way because they merely define abbreviations. Definitions form a conservative extension to the logic, i.e. a sound extension. Other kinds of definitions can be made in the logic which also form conservative extensions. These include definitions of primitive recursive functions, types, and partially defined constants [2]. To guarantee a conservative extension to the logic, the HOL system distinguishes definitions from axioms, and before accepting a new definition, the system first checks that (a) the definition being made is of one of the forms mentioned above, and (b) the constant being defined has not already been defined previously.

A collection of logical types, type operators, constants, definitions, axioms and theorems is called a theory. To make a definition, prove a theorem, or declare a new HOL type, one must first enter a theory and, if facts from other theories are to be used, the relevant existing theories must be declared as parents. For example, if one is working within a theory $t h_{1}$, and an object from theory $t h_{2}$ is required in $t h_{1}$, then $t h_{2}$ must be declared a parent of $t h_{1}$. If $t h_{2}$ is a parent of $t h_{1}$ then all the types, constants, definitions, axioms and theorems available in $t h_{2}$ are available in $t h_{1}$. Thus, $t h_{1}$ is said to be a descendant of $t h_{2}$. Theories, therefore, enable a hierarchical organization of facts. A library of commonly used theories is available in the HOL system to enable the reuse of established and commonly used theorems. The availability of such a library greatly facilitates the task of mechanization and reasoning.

To prove a theorem in a theory, one must apply a sequence of steps (constituting a proof) to either axioms or previously proved theorems by using special ML programs called inference rules. These programs check that the conclusion of a proof follows logically from the hypotheses-in other words, that truth is preserved throughout the proof. For example, given two theorems $\Gamma_{1} \vdash t_{1} \supset t_{2}$ and $\Gamma_{2} \vdash t_{1}$, the inference rule for modus ponens would deduce the theorem $\Gamma_{1} \cup \Gamma_{2} \vdash t_{2}$. The core of the HOL system is made up of a small set of inference rules called primitive inference rules and a small number of definitions and axioms from which all the standard rules of logic are derived. 
The form of proof just described is often referred to as forward proof. One starts with a set of definitions and theorems and manipulates them using the inference rules until the desired theorem to be proved is achieved. The HOL system supports another way of carrying out a proof called goal directed proof or backward proof. The problem with forward proof is that it can often be difficult to foresee which definitions and theorems are required to prove the end result, especially if the proof is long and complicated. The idea of goal directed proof is to do the proof backwards, i.e. start from the desired result (called the goal) and manipulate it until it is reduced to a subgoal which is obviously true. ML functions which reduce goals to subgoals are called tactics and were invented by Milner [24].

The last concept we explain is that of term conversions [24]. A conversion in HOL is a function that maps terms $t$ to theorems expressing the equality of that term with some other term, $\vdash t=u$. Their importance is two-fold: (1) they form the basis for rewriting in HOL, and (2) they facilitate the implementation of inference rules and tactics. Several conversions, and operators for constructing conversions from smaller ones, built into the HOL system (also mostly inherited from the LCF system), played a fundamental role in our mechanization of proof strategies for cos. Examples of the use of some of these conversions are given in later sections.

\section{Mechanizing CCS in HOL}

In this section, familiarity with some of the concepts behind cCS is assumed, so only essential information is presented. We consider a subset of the cos language which does not involve value passing; a subset commonly referred to as pure ccs [23]. The language consists of the inactive process nil, and the operations on processes for denoting prefix (.), summation ( + ), parallel (|), restriction ( () , relabelling ([]), and recursion (rec).

The concrete syntax for pure cCS is given below:

$$
E::=\text { nil }|u . E| E+E|E| E|E \backslash l| E[f]|X| \operatorname{rec} X . E
$$

where $X$ denotes a process variable (which in practice will be bound in a recursive definition), $l$ denotes a visible action called a label, $u$ denotes an action which is either visible (a label) or invisible, i.e. the special action $\tau$, and $f$ denotes a relabelling function on labels.

Labels consist of names and conames where, for any name $a$ say, a coname is written $\bar{a}$. This complement operation on names has the property that $\overline{\bar{l}}=l$, and relabelling conames has the property that $f(\bar{l})=\overline{f(l)}$.

The formal interpretation of each of the above cCS operators is given via an operational semantics [23]. In addition, several behavioral semantics have been defined in terms of bisimulation relations, such as observational [23], trace and testing [9], or branching bisimulation [13]. Each one of these bisimulation relations has an axiomatization which has been proved sound and complete for finite CCS and finite state (pure) CCS. These axiomatizations can be separated into two sets of axioms: (1) those common to all equivalences, referred to as basic axioms, and (2) those which distinguish the various equivalences, referred to as $\tau$-laws.

By means of the basic axioms, any finite ccs term can be proved equivalent to one containing only nil, prefix and summation operators. These basic axioms are shown below:

$$
\begin{array}{ll}
\text { A1: } & E+(F+G)=(E+F)+G \\
\text { A2: } & E+F=F+E \\
\text { A3: } & E+E=E \\
\text { A4: } & E+\text { nil }=E \\
\text { A5: } & \text { nil }[f]=\text { nil } \\
\text { A6: } & (E+F)[f]=E[f]+F[f] \\
\text { A7: } & (u . E)[f]=f(u) \cdot E[f] \\
\text { A8: } & \text { nil } \backslash l=\text { nil } \\
\text { A9: } & (E+F) \backslash l=(E \backslash l)+(F \backslash l)
\end{array}
$$


A10: $\quad(u \cdot E) \backslash l=u \cdot(E \backslash l) \quad$ if $u \notin\{l, \bar{l}\}$, nil otherwise

A11: If $E=\sum_{i \geq 0} u_{i} \cdot E_{i}$ and $F=\sum_{j \geq 0} v_{j} . F_{j}$ then

$$
E \mid F=\sum_{i \geq 0} u_{i} \cdot\left(E_{i} \mid F\right)+\sum_{j \geq 0}^{j \geq 0} v_{j} .\left(E \mid F_{j}\right)+\sum\left\{\tau .\left(E_{i} \mid F_{j}\right) \mid u_{i}=\overline{v_{j}}\right\}
$$

A12: $\quad \operatorname{rec} X . E=E\{\operatorname{rec} X . E / X\}$

where the notation $E\{\operatorname{rec} X . E / X\}$ denotes the substitution of rec $X . E$ for all free occurrences of $X$ in expression $E$.

The axioms which distinguish the various equivalences concern the internal action $\tau$. In this paper we address the theory of observational congruence, so below we only present the $\tau$-laws concerning this theory.

$$
\begin{array}{ll}
\text { T1: } & u \cdot \tau \cdot E=u \cdot E \\
\text { T2: } & E+\tau \cdot E=\tau \cdot E \\
\text { T3: } & u \cdot(E+\tau \cdot F)+u \cdot F=u \cdot(E+\tau \cdot F)
\end{array}
$$

The theory of observational congruence for finite CCS, therefore, is characterized by the axioms A1-A11, T1-T3 shown above.

\subsection{Axiomatizing CCS in $\mathrm{HOL}$}

In HOL, the CCS syntax presented earlier can be mechanized by defining a concrete data type CCS in terms of all its possible constructors. This is done by using a built-in facility for automatically defining concrete recursive data types from their syntactic definition [22]. The input to this definition mechanism is the signature grammar of the operators written in terms of existing types and recursive calls to the type being defined. The type definition for CCS can be done as follows:

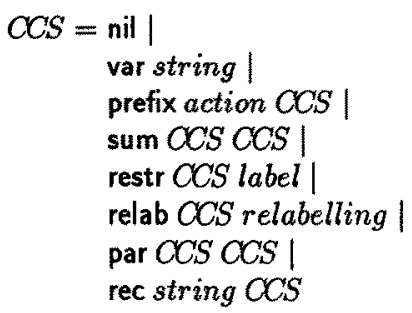

where nil, var, prefix, sum, restr, relab, par and rec are distinct constructors, label and action are syntactic types defined as follows:

$$
\begin{aligned}
& \text { label = const string } \mid \text { compl label } \\
& \text { action = tau } \mid \text { label label }
\end{aligned}
$$

and relabelling is an abbreviation for the function type label $\rightarrow$ label.

To avoid using a verbose prefix notation, and to make the mechanized syntax as similar to that of the CCS language as possible, the parsing and pretty-printing facilities in the HOL system are extended to accept input, and print output, almost identically to that ordinarily associated with cCs. Since the above prefix constructors are therefore only treated internally by the system, we readopt the standard CCS syntax for the rest of the paper except when presenting an example session of using the system in Section 5.

The definition of the data type CCS above only defines the syntax of the language, so now the next steps should be to mechanize the operational semantics of the CCS constructors, define the

\footnotetext{
${ }^{1}$ Modulo ascii syntax, e.g. $\bar{a}$ is written $-a$, and $r$ is written tau.
} 
notion of observational congruence, and derive its axiomatization. There has already been some work to show that it is feasible to mechanize process algebra semantics in HOL and to mechanically prove their axiomatization $[2,3,17]$. Thus, we have decided not to undertake the steps described above for the time being, but to proceed directly to assert the axioms for observational congruence in the HOL logic and to concentrate our efforts on practical reasoning tools at the axiomatic level. We intend to mechanize the semantics and to derive the axioms used in our current level of reasoning later on, to ensure that our mechanization of the CCS theory is also sound.

Having defined the appropriate types and syntactic constructors in the HOL logic, it is, of course, straightforward to assert most of the axioms. The only axioms which require some explanation, in fact, are the expansion laws for the parallel and recursion operators, A11 and A12. We first present the mechanizations of some of the other axioms to illustrate their similarity to their mathematical presentation given earlier, thus demonstrating the suitability of HOL for supporting other notations.

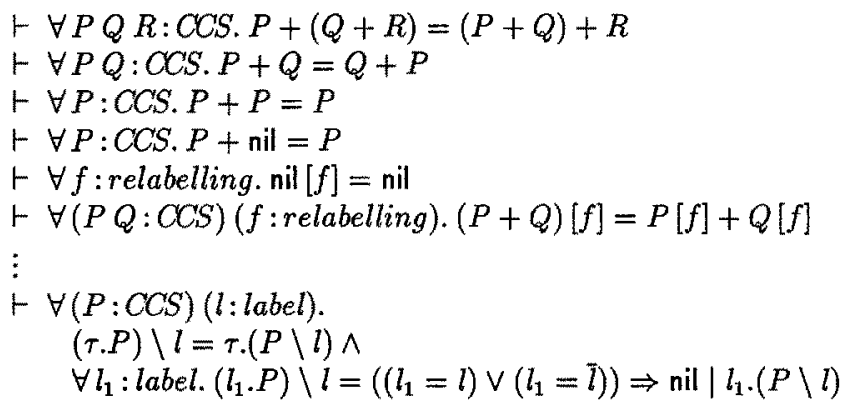

To mechanize axiom A11, we first need to define the notation used for indexed summation. Given an index n:num and a function $P:$ num $\rightarrow C C S$, we define a function SIGMA by primitive recursion such that SIGMA $n P$ denotes the summation $P(0)+P(1)+\ldots+P(n)$.

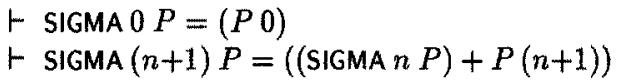

We also need to construct the summation of all processes in a set, so we similarly define a function SET SIGMA as follows:

$\vdash$ SET_SIGMA $\emptyset=$ nil

$\vdash$ SET_SIGMA $(\{x\} \cup X)=x+($ SET_SIGMA $X)$

The axiom for the parallel operator can now be written as follows:

$\vdash \forall i j P Q$.

(SIGMA $i P) \mid($ SIGMA $j Q)=$

(SIGMA $i(\lambda n$. (ACT $(P n)) \cdot((\operatorname{PREF}(P n)) \mid($ SIGMA $j Q))))+$

(SIGMA $j(\lambda n$. (ACT $(Q n)) \cdot((\operatorname{SIGMA} i P) \mid(\operatorname{PREF}(Q n)))))+$

(SET_SIGMA (TAU_SET i $j P Q)$ )

where ACT and PREF are the projection functions on prefixed processes used for extracting the action and the process, respectively:

$\vdash \forall a P, \mathrm{ACT}(a, P)=a$

$\vdash \forall a P \cdot \operatorname{PREF}(a \cdot P)=P$

and TAUSET $i j P Q$ denotes the set of synchronizing processes:

$\vdash \forall i j P Q$.

TAU_SET $i j P Q=$

$\{x \mid \exists n m .(n \leq i) \wedge(m \leq j) \wedge$

$\operatorname{LABEL}(\operatorname{ACT}(P n))=\overline{(\operatorname{LABEL}(\mathrm{ACT}(Q m)))} \wedge$

$x=\tau \cdot((\operatorname{PREF}(P n)) \mid(\operatorname{PREF}(Q m)))\}$ 
The function LABEL is defined merely to project the label from an action, since it is not well-typed to complement an action.

$$
\vdash \forall l \text {. LABEL }(\text { label } l)=l
$$

The above formalization demonstrates that more work than is originally expected can be involved when mechanizing definitions or axioms, because often, axioms written by hand can be packed with notation which itself needs to be mechanized.

The axiom for expanding recursive expressions, A12, is really a meta-axiom in the sense that it is not a single axiom, but a pattern which can be instantiated to any recursive expression to generate particular axioms. We mechanize this in HOL by representing the meta-axiom as a term conversion, i.e. we write a program REC.EXPAND_CONV which, given a recursive expression as a parameter, generates an axiom stating the expansion of the particular recursion. For example, REC_EXPAND_CONV "rec $X . a . X$ " generates the axiom

$$
\vdash \operatorname{rec} X \cdot a \cdot X=a \cdot(\operatorname{rec} X \cdot a \cdot X)
$$

In the following sections we show how the above axiomatization in HOL can be used to reason about cCS specifications.

\section{Reasoning about CCS}

The degree of automation with which one might wish to reason about specifications can vary depending on the complexity of the specifications, and on the level of confidence one has in their correctness. For this reason, we have developed a set of inference rules, tactics and conversions, especially to enable reasoning about CCS expressions by manipulating them according to the axiomatic properties presented in the previous section. The idea is that these rules, tactics, etc., can either be used interactively in a single-step fashion, or they can be composed together to give an automatic strategy.

A wide range of verification strategies can be defined in this way based on the axioms. For example, it is possible to implement a strategy that proves the observational congruence of two finite processes by reducing them to their normal forms, and then by checking if they are equivalent, modulo associativity and commutativity. In fact, we have already implemented strategies for automatically performing some commonly used manipulations, including the one for normal forms.

In this section we describe the implementation of two strategies. The first one explains how a strategy can be defined for using the axioms by adopting selection criteria which depend on the state of the proof, and on the state of the term being manipulated. It computes, fully automatically, the normal form of a finite CCS term with respect to observational congruence and is, in fact, a HOL implementation of the rewriting strategy defined in [19]. The second strategy, on the other hand, is partially interactive; it deals with the expansion laws and shows how it is possible to expand an expression, at the same time keeping its size to a minimum.

\subsection{Normal Forms for Finite CCS}

Before showing the HOL implementation, we first briefly describe the adopted rewriting strategy which, depending on the state of the term, chooses which axiom has to be applied and in which direction, until the normal form is reached. The strategy deals with finite ccs terms containing only nil, prefix and summation operators.

Let Robs denote the term rewriting system obtained by simply directing the axioms $\mathrm{A} 3-\mathrm{A} 4$ and the $\tau$-laws T1-T3 from left to right. Roughly, given a finite summation term, the strategy first reduces the input term with respect to Robs (we refer to this process as Robs_Normalization), and then identifies and deletes summands which are 'semantically contained' in others with respect to observational congruence. To do this, it expands the term using the two $\tau$-laws T2 and T3 (the 
Unfolding step), and at each stage it deletes summands, if any are present, by applying Robs (the Reduction step), until no further summands can be deleted. The normal form of the input term is then obtained by applying those reductions which are exactly opposite to the previous expansions (the Folding step). We can describe this strategy with a regular expression as follows:

$$
\text { strat }=\text { Robs.Normalization; Absorption }
$$

where

$$
\text { Absorption }=(\text { Unfolding; Reduction })^{*} ; \text { Folding }^{*}
$$

We do not give details of the mechanization of the strategy in HOL but merely illustrate the implementation, as a HOL conversion, of strat shown above.

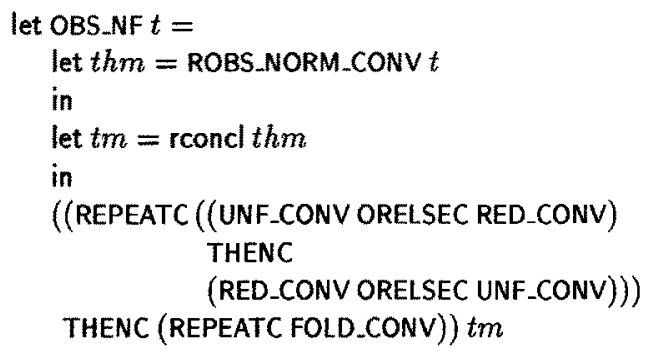

where rconcl is a function which returns the right hand side expression of the conclusion of an equality theorem, ROBS_NORM_CONV, UNF_CONV, RED_CONV and FOLD_CONV are the conversions related to the basic steps of the strategy, and REPEATC, THENC and ORELSEC are built-in conversions with the obvious meanings. In the above program, given an input term $t, t h m$ is the theorem $\vdash t=t m$ returned by ROBS_NORM_CONV, where the term $t m$ is the normal form of $t$ with respect to Robs, and is input to the compound conversion which mechanizes the Absorption step.

\subsection{A Lazy Expansion Strategy}

One of the main problems in the verification of interleaving specifications of concurrent processes is state explosion: the number of processes in a system potentially increases exponentially in the number of its parallel components. For verification environments based on a finite state representation of processes, this can constitute a big limitation because it makes even processes whose structure is in principle very simple, unmanageable. As discussed in [18], the state space of a complete system often contains a number of computations that are hidden at the global level, and thus irrelevant to its external behaviour. Global context conditions do not permit the construction of the minimal process representation by minimizing the single components. Attempts to control state explosion have been proposed [18] which are able to reduce the problem by requiring the user to supply additional information on the way subsystems interact.

In our framework, there is a rather natural way to cope with this problem. This is based on the fact that single step transformations corresponding to individual axioms can be performed to allow the manipulation of intermediate terms. The strategy, in fact, is the one generally used to verify a system by hand: to first apply an expansion step, and then to reduce the resulting expression as much as possible by using other appropriate axioms. In this way it is possible to eliminate all irrelevant components as soon as they are produced. For example, consider the following simple term:

$$
\left(\mu_{1}, P_{1}\left|\mu_{2}, P_{2}\right| \overline{\mu_{2}} \cdot P_{3}\right) \backslash \mu_{1} \backslash \mu_{2}
$$

After one expansion step, a new term is obtained composed of four summands. It becomes immediately clear that by using the simplification axioms for restriction, A8-A10, three of the four 
summands are reduced to nil, and these can in turn be eliminated by means of the axiom for deleting nil summands, A4. The expression is thus reduced to the $\tau$ prefixed one

$$
\tau \cdot\left(\left(\mu_{1} \cdot P_{1}\left|P_{2}\right| P_{3}\right) \backslash \mu_{1} \backslash \mu_{2}\right)
$$

where only one parallel composition requires further expansion, instead of the original four.

In the following section, we show how this simple strategy is used to prove the equivalence between a specification and an implementation of a simple scheduler. In the example it is shown that when dealing with recursive processes, the expansion step might remain applicable indefinitely. Thus, the issue of controlling the termination of the expansion law has to be considered. An automatic solution to this problem is possible only when dealing with finite state processes, and at the expense of recording all the intermediate terms computed along the proof. This problem, therefore, is one which will benefit from interactive facilities.

\section{Example: Verification of a Simple Scheduler}

Below we illustrate how the mechanization of cCs described in the preceding sections is used to reason about CCS specifications by presenting transcripts of a HOL session. The example we consider is that of a simple scheduler shown in Figure 1 where two processes, a reader and a writer, contest via a semaphore to perform their tasks.

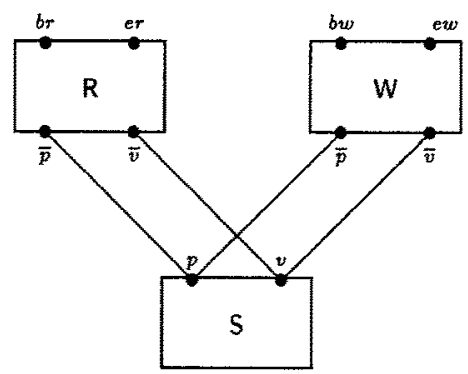

Figure 1: Implementation of a Scheduler

One interacts with the HOL system via the programming language ML. The ML prompt is *, so lines beginning with * show text typed by the user, and other lines show the system's response. The user's input is always terminated by two successive semi-colons. Terms in the HOL logic are distinguished from ML expressions by enclosing them in double quotes. To help readability, the HOL transcripts are edited to show proper logical symbols instead of their ascii representations.

We begin by entering a theory (call it scheduler) in which we reason about the scheduler, and we declare the mechanized theory for cos described earlier (call it ccs) as a parent of this theory. To do this we invoke the built-in ML functions new_theory and new-parent in sequence, both of which return the value () of ML type void to indicate success.

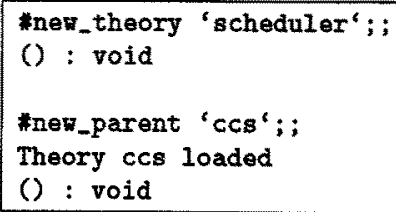

We begin by defining the behaviour of the components of the scheduler. The reader, $R$, makes a request to begin reading. If the request is acknowledged, then the reader performs its reading 
task, and when finished it signals back to the semaphore. The reader is represented in CCS by a process $\mathrm{R}$ say, which recursively performs a request action -' $\mathrm{p}$ ', followed by a begin-read action 'br', an end-read action 'er', and a terminating signal '' $v$ '. The behaviour of the writer, $W$, and its specification $\mathrm{w}$, are identical, but with writing actions replacing the reading ones. The two specifications are defined in HOL below by invoking the ML function new_definition.

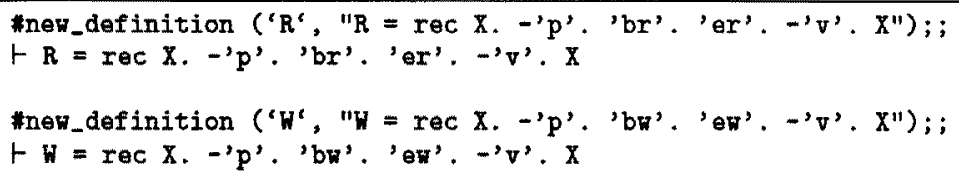

The semaphore scheduler $S$ can be simply specified as a process $S$ which recursively performs the synchronizing actions ' $p$ ' and ' $v$ '.

\#nen_definition (' $\mathrm{s}$ ', "S = rec $X$. ' $p$ '. ' $v$ ', $X ")$; ;

$\vdash s=\operatorname{rec} X$. ' $p$ '. 'v'. $X$

The three components put together can now be specified by composing the processes in parallel, and hiding the internally synchronizing actions ' $p$ ' and ' $v$ '.

"new_definition ('Impl', "ImpI = (R | S | W) | 'p'|'v'"); ;

$t$ Impl $=(R|S| W) \mid$ 'p' $\mid$ 'v'

We would now like to prove that this implementation of the scheduler meets the specification that expresses the property of recursively engaging in either reading or writing, i.e. that

$$
\text { Impl = tau. 'bw'.'ew'.Impl + tau.'br'.'er'.Impl }
$$

To prove this, we use various conversions implemented in the system for manipulating ccs specifications. We begin by expanding the definition for Impl, and then expanding those for $R, W$ and s.

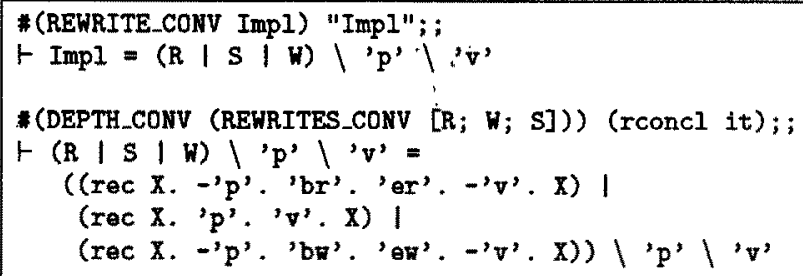

In the session above, it is a built-in ML variable which is automatically updated by the system to refer to the last computed value, and the function rconcl, as explained in Section 4.1 , returns the right hand side expression of the conclusion of an equality theorem. The conversion functional DEPTH_CONV applies a given conversion to every applicable sub-term of a term.

The next step in the proof is to expand the each of the recursive expressions once, by means of the conversion REC.EXPAND_CONV described in Section 3.1, and then to repeatedly expand-and-simplify the parallel composition expressions as described in the previous sections. These steps each produce rather long intermediate theorems, so we do not present them here. In fact we skip the presentation of four successive expansions and simplifications of the parallel compositions, done via a conversion called NORM_conv, to reach the stage when the original recursive expressions are recognized, as shown in the session below. 


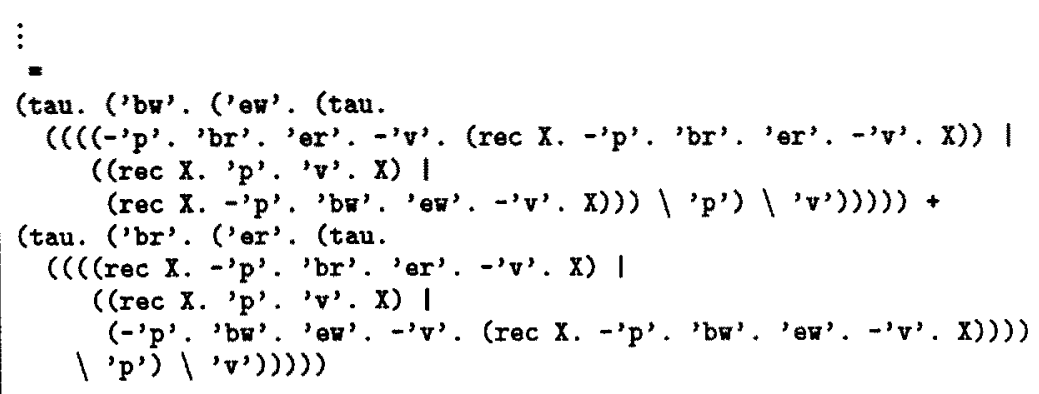

Two immediate simplifications can be noted from the expression above. The first involves the elimination of redundant $r$ 's according to the axiom $\mathrm{T} 1$. The second simplification is based on recognizing that the remaining parallel compositions have returned back to their original form, modulo two expanded recursive components. We can now, therefore, simplify the remaining expression by folding back the expanded recursive definitions. Clearly it is essential to recognize this 'loop' in the course of the proof since otherwise, one could go on forever expanding recursive expressions and using the expansion law for parallel composition. As mentioned in the previous section, it is possible to implement strategies for automatically detecting when to stop such expansions for certain classes of expressions. In this case, however, we rely on user intervention.

Below we show the final stages of the proof by combining the steps for eliminating redundant $\tau$ 's, folding back the expanded recursive expressions, and collapsing back the definitions for $\mathrm{R}, \mathrm{S}, \mathrm{W}$ and Impl.

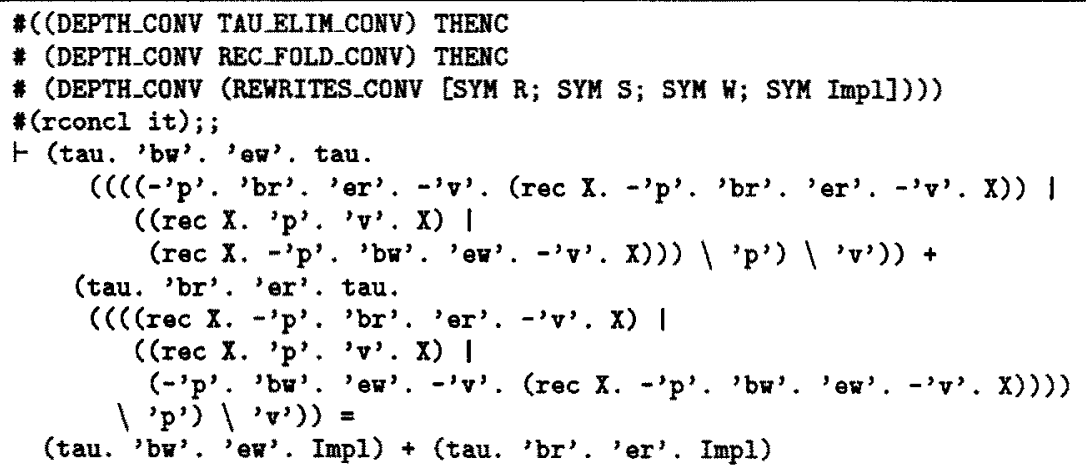

By the law of transitivity, we have proved the intended theorem. Of course, as many of the involved proof steps as desired can be combined, as shown above, to control the size of the proof step to be undertaken, i.e. to control the extent of automation in the reasoning process.

\section{Related Work}

As mentioned in the introduction, several verification systems for process algebras already exist, and most of these systems rely on a finite state machine representation of specifications. In this section we give a brief overview of some of these systems, and discuss them in relation to our approach.

Verification systems based on finite state machine representations, e.g. Auto [11], the Concurrency Workbench [6], Aldebaran [12], and Squiggles [1], implement some rather efficient automatic algorithms for process algebra verification. They have, however, a few limitations, mostly due to the fact that they do not provide sufficient control over the verification process. Their problems with state explosion could be significantly reduced if the analysis of the state machines could be 
controlled because in most cases many states can be pruned, and the state space optimised, to yield a manageable system. Of course, a completely automatic approach can only deal with finite state processes, and there is no way, in such frameworks, to accommodate the verification of infinite state processes, even though the theory behind the process algebras supports such reasoning.

Another drawback for such systems is that they do not, in general, provide good diagnostics to help identify errors. The TAV system [14], based directly on the definition of bisimulation, permits improved diagnostic features, but at the expense of reduced efficiency. It is, in fact, able to provide the user with logic formulae which distinguish between specifications if they are not bisimilar.

We hope that the approach we have adopted will help solve some of the problems of fully automatic systems. Our research stems from two separately developed pieces of work, described in [10] and [3]. In [10], a kernel specification system for a subset of CCS is presented. The system is based on the axiomatic presentation of behavioural equivalences, and relies on the use of rewriting techniques. The basic idea of the system is to use the term representation and axiomatization of CCS specifications, and to allow the definition of automatic strategies in addition to those already built-in, much in the same philosophy to the ideas described in this paper. It was felt, however, that a rewriting system alone would be quite restrictive, and that a system with a meta-language to facilitate programming additional strategies, as well as provide more general and powerful proof techniques, was required. The mechanization of the CSP failure-divergence semantics in HOL described in [3], suggested that HoL might be a suitable environment for supporting process algebras in the manner we are advocating, so the work described in this paper was intended as a first attempt at investigating this.

Other approaches directly related to ours exist. These include an investigation by Cleaveland and Panangaden into mechanizing CCS using the NUPRL logical theory of types [5], a proof tool for CSP developed using the B-tool [8], an ongoing mechanization of Milner's $\Pi$-calculus in HOL being undertaken by Gordon and Melham [17], and the development of an axiomatic rewriting tool for cCS being undertaken at the University of Sussex [21]. All the above efforts, however, have been almost totally investigatory so far, and more research into mechanizing specialised formalisms using different embedding logics and proof systems is desired if this approach is to demonstrate it can be used in a natural and practical way for verifying process algebra specifications.

\section{Conclusion}

In this paper we have presented an attempt to use the HOL theorem prover as a verification environment for reasoning about cCs specifications. The purpose of this work has been to provide evidence that mathematical proof techniques, based on the axiomatic representation of process algebras, provide a promising approach to the mechanical verification of concurrent systems. We believe that such a system can be used practically, only if it offers an environment in which reasoning can be conducted both automatically and interactively.

Due to the facilities for modularity in HOL, it is possible to mechanize various behavioural semantics, and to derive axiomatizations and proof tools for them. In the paper we have neither used, nor mentioned, most of the facilities Hou provides which we hope to exploit in our forthcoming work. Among these, are the type system which we hope will enable the incorporation of data with processes, as in value passing, and the facility for performing proofs by induction which will facilitate reasoning about indexed processes.

Future work will mainly be devoted to extend the functionalities of the HOL-CCS environment. For example, we hope to provide a program for automatically terminating successive applications of the lazy-expansion strategy for parallel composition, and some smart criteria for automatically selecting which parallel expression to expand first. In fact, we have already begun to extend the strategy for computing the observational normal forms for finite CCS expressions (explained earlier) to deal with expressions with finite state processes, in the way described in [20]. Of course, we also intend to step back and mechanize the operational semantics of CCS, define the various notions of 
equivalence and congruence that exist for cos (including the observational one described in this paper), and to mechanically derive the respective axiomatizations from their semantics.

\section{Acknowledgements}

We are grateful to Rocco De Nicola of IEI-CNR for his technical advice and continuous encouragement, and to Robin Gallimore and Roger Fleming of Hewlett-Packard Laboratories for supporting this work. Thanks are also due to the management of the Hewlett-Packard Pisa Science Centre for providing the opportunity to carry out this collaboration.

\section{References}

[1] Bolognesi T., Caneve M., 'Squiggles-A Tool for the Analysis of LoTos Specifications', in Formal Description Techniques, K. Turner (ed.), North-Holland, 1989.

[2] Camilleri A. J., 'Mechanizing CsP Trace Theory in Higher Order Logic', IEEE Transactions on Software Engineering, Special Issue on Formal Methods, N. G. Leveson (ed.), September 1990, Vol. 16, No. 9., pp. 993-1004.

[3] Camilleri A. J., 'A Higher Order Logic Mechanization of the CSP Failure-Divergence Semantics', in proceedings of the 4th Higher Order Workshop, Banff, Springer-Verlag (to appear).

[4] Church A., 'A Formulation of the Simple Theory of Types', The Journal of Symbolic Logic, 1940 , Vol. 5 , pp. 56-68.

[5] Cleaveland R., Panangaden P., 'Type Theory and Concurrency', International Journal of Parallel Programming, November 1988, Vol. 12, No. 2, pp. 153-206.

[6] Cleaveland R., Parrow J., Steffen B., 'The Concurrency Workbench: Operating Instructions', Technical Note LFCS-TN-10, University of Edinburgh, Laboratory for the Foundations of Computer Science, September 1988.

[7] Cousineau G., Huet G., Paulson L., 'The ML Handbook', INRIA, 1986.

[8] Davies J., 'Assisted Proofs for Communicating Sequential Processes', M.Sc. Dissertation, Oxford University Computer Laboratory, Programming Research Group, September 1987.

[9] De Nicola R., Hennessy M. C., 'Testing Equivalence for Processes', Journal of Theoretical Computer Science, 1983, Vol. 34, pp. 83-133.

[10] De Nicola R., Inverardi P., Nesi M., 'Using Axiomatic Presentation of Behavioural Equivalences for Manipulating ccs Specifications', Proceedings of the Workshop on Automatic Verification Methods for Finite State Systems, Grenoble, France, June 12-14, 1989, Lecture Notes in Computer Science, Springer-Verlag, 1990, Vol. 407, pp. 54-67.

[11] De Simone R., Vergamini D., 'Aboard AUTO', Technical Report 111, INRIA, 1989.

[12] Fernandez J., C., 'Aldebaran: Un système de vérification par réduction de processus communicants', Ph.D. Thesis, Université de Grenoble, 1988.

[13] van Glabbeek R.J., Weijland W.P., 'Branching Time and Abstraction in Bisimulation Semantics', Proceedings of the IFIP 11th World Computer Congress, San Francisco, 1989.

[14] Godskesen J.C., Larsen K.G., Zeeberg M., 'TAV Users Manual', Internal Report, Ålborg University Centre, Denmark, 1989. 
[15] Gordon M. J. C., 'HOL-A Proof Generating System for Higher-Order Logic', in VLSI Specification, Verification and Synthesis, Birtwistle G. and Subrahmanyam P. A. (eds.), Kluwer Academic Publishers, Boston, 1988, pp. 73-128, Proceedings of the Hardware Verification Workshop, Calgary, Canada, 12-16 January 1987.

[16] Gordon M. J. C., 'Mechanizing Programming Logics in Higher Order Logic', in Current Trends in Hardware Verification and Automated Theorem Proving, Birtwistle G., Subrahmanyam P. (eds.), Springer-Verlag, 1989, pp. 387-439, Proceedings of the Banff Workshop on Hardware Verification, Banff, Canada, 1988.

[17] Gordon M., Melham T., 'Mechanizing the II-calculus in HOL', Private Communication, 1990.

[18] Graf S., Steffen B., 'Compositional Minimization of Finite State Processes' Proceedings of the Workshop on Computer-Aided Verification, New Brunswick, New Jersey, June 1990.

[19] Inverardi P., Nesi M., 'A Rewriting Strategy to Verify Observational Congruence', Information Processing Letters, 1990, Vol. 35, pp. 191-199.

[20] Inverardi P., Nesi M. 'Deciding Observational Congruence of Finite-State ccs Expressions by Rewriting', Internal Report B4-10, Istituto di Elaborazione dell' Informazione, C. N. R., March 1990.

[21] Lin H., 'A Process Algebra Manipulator', Technical Report, University of Sussex Computer Laboratory, (to appear).

[22] Melham T. F., 'Automating Recursive Type Definitions in Higher Order Logic', in Current Trends in Hardware Verification and Automated Theorem Proving, Birtwistle G., Subrahmanyam P. (eds.), Springer-Verlag, 1989, pp. 341-386, Proceedings of the Banff Workshop on Hardware Verification, Banff, Canada, 1988.

[23] Milner R., Communication and Concurrency, Prentice-Hall Int., 1989.

[24] Paulson L. C., Logic and Computation-Interactive Proof with Cambridge LCF, Cambridge Tracts in Theoretical Computer Science (2), Cambridge University Press, 1987. 